\title{
The nature and titer of juvenile hormone in the brown planthopper, Nilaparvata lugens (Homoptera: Delphacidae) in relation to wing morphogenesis and oocyte development
}

\author{
Arlene G. Bertuso and Sumio Tojo* \\ Laboratory of Applied Entomology, Department of Applied Biological Sciences, Faculty of Agriculture, Saga University, Saga \\ 840-8502, Japan
}

(Received 23 August 2001; Accepted 29 October 2001)

\begin{abstract}
Whole body extracts from nymphs and adults of the brown planthopper, Nilaparvata lugens were analyzed for the known insect juvenile hormones (JHs) by gas chromatography-mass spectrometry (GC-MS) with selected ion monitoring. JH was separated successively by solvent extraction, chromatography on an aluminum oxide column and high performance liquid chromatography. JH III was identified as the only type of JH in both stages of this species. As the levels were very low for quantitative determination of JH III by GC-MS, we prepared haemolymph juvenile hormone binding protein (JHBP) of Locusta migratoria which had a high affinity for JH III and used it in a competitive binding assay to quantify JH III in the haemolymph of the brachypterous and macropterous pure lines of $N$. lugens. However, the JH III titer developmental profiles were similar in the brachypterous and macropterous fifth (final) stadium nymphs. JH III titers in the haemolymph of the brachypters were significantly higher than those of macropters, coinciding with earlier development of oocytes in the brachypters, which supported the regulation of ovarian development by JH III.
\end{abstract}

Key words: Juvenile hormone III, gas chromatography-mass spectrometry, wing-form, ovarian development, Nilaparvata lugens

\section{INTRODUCTION}

Juvenile hormone ( $\mathrm{JH})$ plays an important role in the regulation of insect metamorphosis. It also contributes to many physiological and behavioral aspects of insect development, such as vitellogenin synthesis and ovarian development in adults, diapause, phase differentiation, caste determination in social insects, and other aspects of metabolism associated with these functions (Nijhout, 1994). Utilization of methods such as gas-liquid chromatography with electron capture detection (Bergot et al., 1976), gas chromatography or liquid chromatography coupled with mass spectrometer detection (Mauchamp et al., 1979; Bergot et al., 1981; Rembold and Lackner, 1985), radioimmunoassay (Strambi et al., 1984) and competitive protein binding assay (Goodman et al., 1976; Glinka et al., 1995) paved the way for numerous studies on $\mathrm{JH}$ titer determinations at physiological levels in a variety of insect species. To date, five structurally related $\mathrm{JH}$ have been identified in insects (reviewed by Davey, 2000; Gilbert et al., 2000) with the addition of $\mathrm{JHB}_{3}$, methyl farnesoate (Riddiford, 1994; Yin et al., 1995) and two hydroxyl JH derivatives (Darrouzet et al., 1997). In the order Hemiptera (suborder Homoptera), little is known regarding the endocrine mechanisms involving the regulation of metamorphosis and reproduction in this group of insects, and even less is known about the chemical identity and titer regulation of the JH. Their small size makes them difficult subjects for experimental endocrinology. However, indirect observations from exogenous applications of $\mathrm{JHs}$ and $\mathrm{JH}$ analogue (JHA) have begun to provide some insight into the nature of JHs in this order (reviewed by Staal, 1975, 1986). In the study conducted on Oncopeltus fasciatus, by Rankin and Riddiford (1977, 1978) reported that this bug released a precursor, methyl farnesoate, rather than its final product (i.e., JH III in vitro). Using different incubation medium, Bowers et al. (1983) extracted JH III as the products released by the corpora allata (CA) of Dysdercus fasciatus, Nezara viridula and $O$. fascia-

* To whom correspondence should be addressed at: E-mail: tojos@cc.saga-u.ac.jp 
tus. Numata et al. (1992) detected the presence of JH I from the haemolymph of the bean bug, Riptortus clavatus. However, Kotaki (1993, 1996) reported that neither $\mathrm{JH}$ I, JH III nor $\mathrm{JHB}_{3}$ were released in vitro by the CA of this species. The first identification of $\mathrm{JH}$ in Hemiptera was that of Hardie et al. (1985) in which JH III (mean low value of only $\sim 0.15 \mathrm{ng} / \mathrm{g}$ ) was found to be the predominant JH in Megoura viviae and Aphis fabae.

In the brown planthopper, Nilaparvata lugens, no information has been published on the chemical identity and titer regulation of the JH. Evidence that $\mathrm{JH}$ plays an important role in the development of $N$. lugens comes from studies in which exogenously applied JHs and JHA have been shown to affect metamorphosis and wing morphogenesis (Wilkins and Sim, 1983; Iwanaga and Tojo, 1986; Ayoade et al., 1996a, b, 1999). In addition to the role of $\mathrm{JH}$ during nymphal growth and metamorphosis, JHs are also known to affect ovarian development in female planthoppers (Iwanaga and Tojo, 1986; Ayoade et al., 1999). Information on the nature and titer of $\mathrm{JH}$ should be useful in understanding the role of $\mathrm{JH}$ in the developmental processes of $N$. lugens.

\section{MATERIALS AND METHODS}

Insects. Studies were performed on $N$. lugens maintained in acryl-resin cylinders $(5.4 \mathrm{~cm}$ in diameter $\times 22.0 \mathrm{~cm}$ in height) containing ca. 130 rice seedlings (variety Reiho) under a $16 \mathrm{~h}$ light: $8 \mathrm{~h}$ dark photoregime at $25 \pm 2^{\circ} \mathrm{C}$ (Morooka and Tojo, 1992). N. lugens nymphs were transferred to new cylinders with fresh rice seedlings $4,13-15$ and 21-24 d after hatching. To synchronize the development of the respective nymphal instars, newly ecdysed individuals were removed from the colonies every $6 \mathrm{~h}$ and placed in other cylinders. In this study, batches of insects (between 1.0 and $1.6 \mathrm{~g}$ ) from different nymphal and adult stages of $N$. lugens were analyzed for the known JHs. JH titers in the haemolymph were measured in the unmated macropterous and brachypterous pure lines of this species staged at daily intervals after eclosion. The brachypterous and macropterous pure lines of $N$. lugens used in this study were selected under highly crowded conditions over 100 generations for the short-wing and long-wing forms, respectively (Morooka and Tojo, 1992).
Dissections. Twenty five brachypterous and macropterous females were collected on $1,2,3$ or $4 \mathrm{~d}$ after adult emergence. The abdomens from all females were immediately frozen at $-20^{\circ} \mathrm{C}$ for later dissection. After thawing, the two ovaries were removed and placed in $0.9 \%$ saline. Several ovaries were randomly selected and their terminal oocytes were measured under a dissecting microscope equipped with an ocular micrometer.

Chemicals. All chemicals used in this study were of at least reagent grade and all solvents were of at least HPLC grade. Juvenile hormone III (JH III) was purchased from Sigma Chemical Company, while $\left[{ }^{3} \mathrm{H}\right] \mathrm{JH}$ III $(481.06 \mathrm{~Bq} / \mathrm{mmol})$ was obtained from New England Nuclear Co.

Extraction and purification of JH from whole animals. Whole body samples of 1.0 to $1.6 \mathrm{~g}$ nymphs or adults of $N$. lugens were placed in a $10 \mathrm{ml}$ glass homogenizer containing $0.5 \mathrm{ml}$ of icecold acetonitrile. JH extractions using insect tissues were processed by the method of Bergot et al. (1981). Elution of hormones was performed on $1.0 \mathrm{~g}$ aluminum oxide column (activity grade III) using $2 \mathrm{ml}$ diethyl ether. Eluents were collected, evaporated, and dissolved in methanol for HPLC purification.

High performance liquid chromatography (HPLC). Final purification of JHs was done according to the method of Mauchamp et al. (1979). $\mathrm{JH}$ was purified on a liquid chromatograph (Gilson) with a CAPCELL PAK $\mathrm{C}_{18}$ (AG 120) column, $250 \times 4.6 \mathrm{~mm}$ (Shiseido), and eluted with $80 \%$ methanol in water at a flow rate of $1 \mathrm{ml} / \mathrm{min}$. Injection was performed using a Rheodyne 7125 injector, while the UV detection was monitored at $215 \mathrm{~nm}$. Purified JH fractions were collected, evaporated, and dissolved in acetonitrile for gas chromatography-mass spectroscopic (GC-MS) analysis.

Gas chromatography-mass spectrometry. JH analysis by gas chromatography-mass spectrometry (GC/MS) was performed on a Hewlett Packard (5890 Series II Plus) gas chromatograph coupled with a Hewlett-Packard GC/MS Selective Ion Detector equipped with a data acquisition system. The $\mathrm{JH}(\mathrm{s})$ was resolved on a GC capillary column $(60 \times$ $0.25 \mathrm{~mm}$ ). Several different stationary phases were used during the course of the investigations: method A: $1 \%$ OV 80 on an INNOWax, column programmed from 40 to $220^{\circ} \mathrm{C}$ at a rate of 3 to $5^{\circ} \mathrm{C}$ per min; method B: $1 \%$ OV 180 on INNOWax, col- 
umn programmed from 40 to $220^{\circ} \mathrm{C}$ at a rate of 3 to $5^{\circ} \mathrm{C}$ per min; method $\mathrm{C}: 0.5 \%$ FFAP 60 on an INNOWax, column programmed from 200 to $400^{\circ} \mathrm{C}$ at a rate of $7^{\circ} \mathrm{C}$ per min. Effective $\mathrm{JH}(\mathrm{s})$ separation and detection were achieved when $1 \%$ OV 180 on an INNOWax column was used in GC/MS.

JH binding assay for haemolymph of JH. All glassware used in $\mathrm{JH}$ binding was pre-coated with $10 \%$ polyethylene glycol (PEG, MW 20,000), rinsed, and dried at $45^{\circ} \mathrm{C}$ (Goodman et al., 1976). $\mathrm{JH}$ binding protein (JHBP) prepared from the haemolymph of Locusta migratoria treated with $500 \mu \mathrm{g}$ ethoxyprecocene (Chinzei et al., 1982) was purified according to the method described by Glinka et al. (1995). JHBP concentration was determined by the DC assay (BioRad DC Protein Assay, U.S.A.) using bovine serum albumin (BSA) as a standard. The JHBP solution was lyophilized after dialysis against $0.2 \%$ ammonium bicarbonate.

Haemolymph samples $(20-40 \mu \mathrm{l})$ from $N$. lugens $(n=40-188)$ were collected and processed for binding assay based on the combined methods of Strambi et al. (1984) and Glinka et al. (1995). JH from the haemolymph sample was eluted from a Sep-Pak silica cartridge with $1.5 \mathrm{ml}$ hexane : ether (9:1 by volume), evaporated with a stream of nitrogen and dissolved in an assay buffer $(10 \mathrm{~mm}$ Tris-HCl pH 8.0, $100 \mathrm{~mm} \mathrm{KCl,} 1$ mm EDTA, 10\% glycerol, $100 \mu \mathrm{g} / \mathrm{ml} \mathrm{BSA)} \mathrm{by} \mathrm{sonication} \mathrm{for} 10$ $\min$.

$\mathrm{JH}$ binding was estimated in borosilicate glass tubes $(10 \times 75 \mathrm{~mm})$ containing $200 \mu \mathrm{l}$ assay buffer and $100 \mu \mathrm{l}$ JHBP pre-incubated with $\left[{ }^{3} \mathrm{H}\right] \mathrm{JH}$ III (about $8,000 \mathrm{dpm}$ ) for $30 \mathrm{~min}$ at $25^{\circ} \mathrm{C}$. Standards of $\mathrm{JH}$ III from 0 to $3.5 \mathrm{pM}$ and $50 \mu \mathrm{l}$ samples of haemolymph extract processed as mentioned above, were prepared in the assay buffer and $100 \mu \mathrm{l}$ of each of these solutions was added to the assay tubes. A $30 \mathrm{~min}$ incubation period at $25^{\circ} \mathrm{C}$ was allowed for exchange reaction. JH bound to JHBP was separated from free hormone with $0.5 \mathrm{ml}$ of 5\% hydroxyapatite (HAP) suspension, as described by Roberts and Wyatt (1983). Radioactivity was measured in the pelleted precipitate on glass fiber filters (Whatman GF/A or Whatman 934-AH) and supernatant, after overnight incubation with scintillation fluid (Ultima Gold MV, Packard), with a Wallac 1410 Liquid Scintillation Counter. Radioactivity in the precipitate represented JHBP-bound-
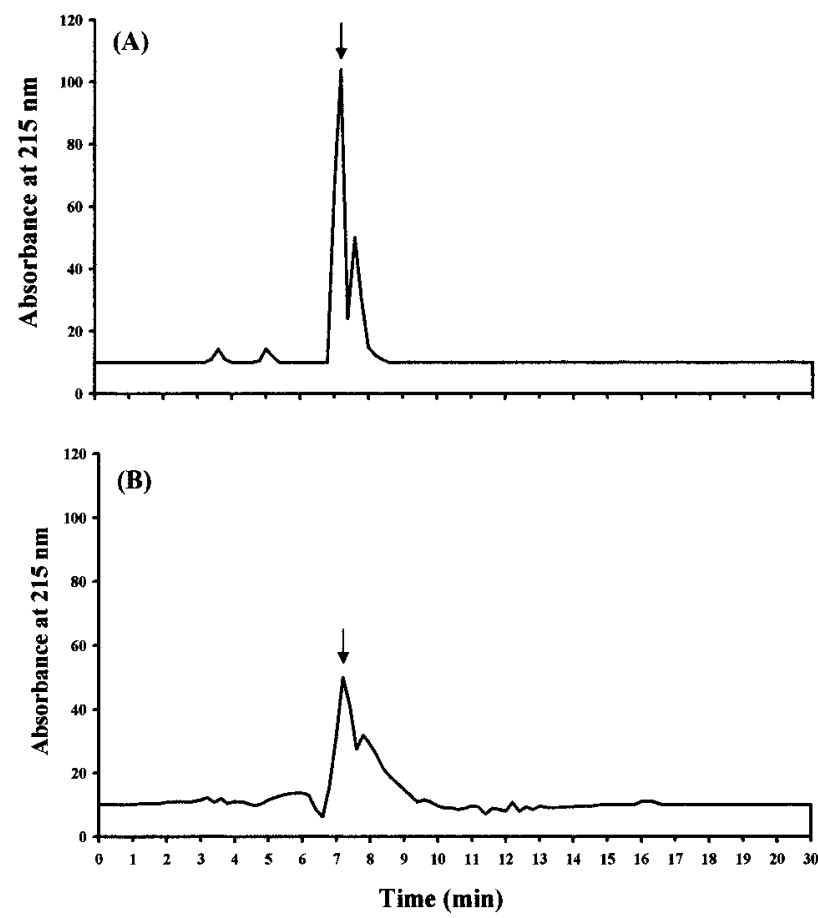

Fig. 1. HPLC separation of JH III and JH extract from whole body of $1.16 \mathrm{~g}$ adult $N$. lugens. (A) standard JH III; (B) insect extract. The arrows indicate the elution times of radioactive JH III. HPLC conditions: CAPCELL PAK $\mathrm{C}_{18}$ (AG 120) column, $250 \times 4.6 \mathrm{~mm}$, effluent $80 \%$ methanol in water, flow rate of $1 \mathrm{ml} / \mathrm{min}$.

$\mathrm{JH}$, while radioactivity in the supernatant represented free ligand.

\section{RESULTS}

\section{Identification of $\mathbf{J H}$}

As shown in Fig. 1, HPLC on a CAPCELL PAK $\mathrm{C}_{18}$ column separated a modest peak corresponding to JH III (retention time 7.0 to $7.2 \mathrm{~min}$ ) when whole body extracts from $1.16 \mathrm{~g}$ adults were subjected to the column. Further analysis of the major JH peak in HPLC by GC-MS in a selected ion mode revealed a peak at a retention time of 15.43 min. The mass-spectrum of the major peak corresponding in retention time to JH III in $N$. lugens extracts obtained by HPLC coincided with that of JH III, with fragment ions $m / z 267,249$ and 235 (Fig. 2e). Thus, the presence of JH III was confirmed, but other known JHs could not be detected by GC-MS. Selected ion monitoring of the fragment ions $m / z$ 267, 249 and 235 from $N$. lugens extract revealed a clear peak corresponding to JH III in the retention time (Fig. 2). The presence of $\mathrm{JH}$ 
$\mathbf{a}$

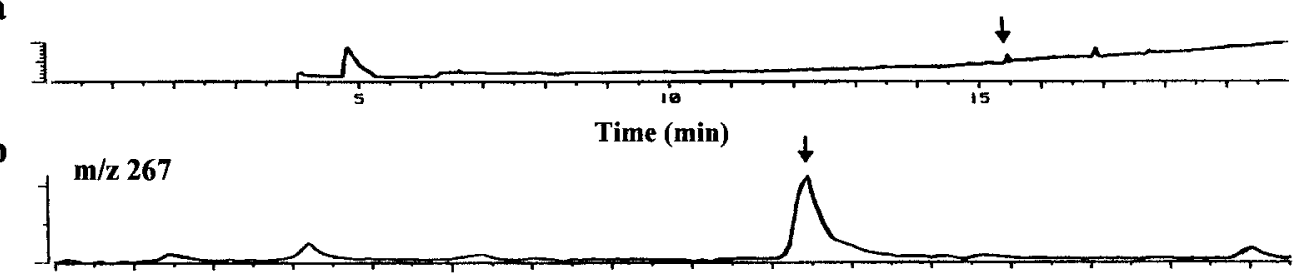

c

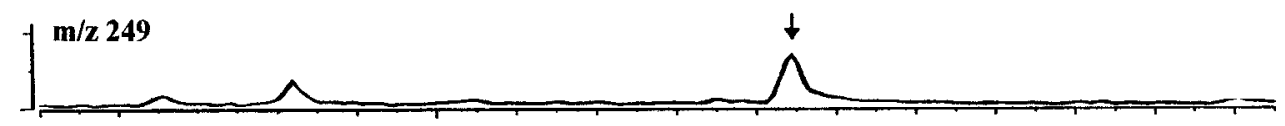

d

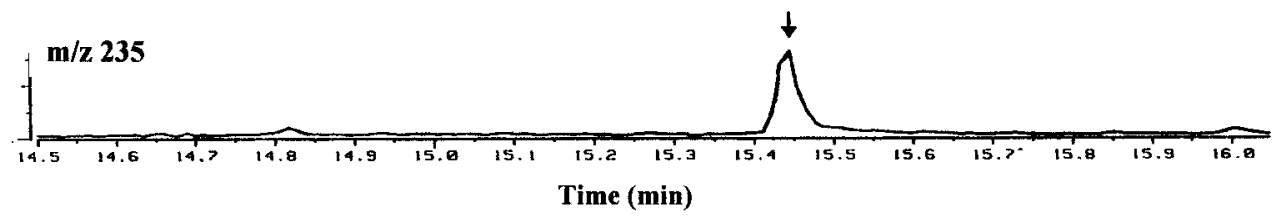

$\mathbf{e}$

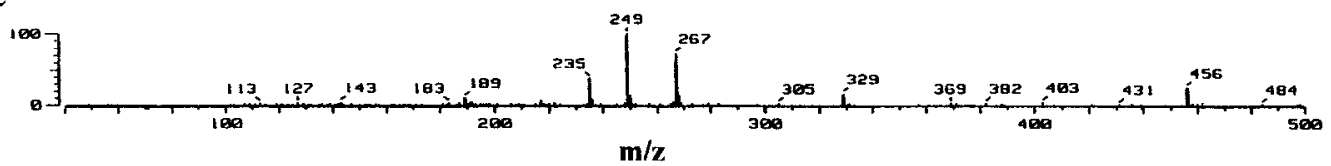

Fig. 2. Separation of the major peak component separated by HPLC of the extract from whole body of adult $N$. lugens (Fig. 1) on an INNOWax capillary column $(60 \mathrm{~mm} \times 0.25 \mathrm{~mm}$ I.D.) (a), and selected ion monitoring chromatographs in GC-MS analysis for $m / z 267$ (b), 249 (c), 235 (d) and in the mass spectra of the same sample after ionization by ammonium. Mass spectra of standard $\mathrm{JH}$ III is presented in the bottom figure (e).

III was also confirmed in whole body extract from fifth stadium nymphs (data not shown). Although JH III has been detected in $N$. lugens, the relatively low abundances of ions $\mathrm{m} / \mathrm{z} 267,249$ and 235 made it difficult to calculate the exact amount of JH III.

\section{Standardization of the competition assay for $\mathrm{JH}$ III determination}

The suitability of the haemolymph JHBP of $L$. migratoria for use in a competition assay for JH III has been investigated. To optimize the assay conditions for maximum sensitivity, partially purified JHBP was serially diluted and assayed for binding activity (Fig. 3). A dilution which bound about $30-40 \%$ (about $2.1-2.6 \mu \mathrm{g} / \mu 1$ protein) of the total amount of the labeled ligand $\left({ }^{3} \mathrm{H}\right] \mathrm{JH}$ III) was then used for the assay. The amount of $\left[{ }^{3} \mathrm{H}\right] \mathrm{JH}$ III bound was found to be unchanged when the exchange reaction was carried out for periods ranging from $1 \mathrm{~min}$ to $9 \mathrm{~h}$ (data not shown). Therefore, all incubations were carried out for $30 \mathrm{~min}$ at $25^{\circ} \mathrm{C}$.

A standard curve based on various concentrations of unlabeled racemic JH III ranging from 0 to $3.5 \mathrm{pm}$ was constructed for each assay (see Fig. 4

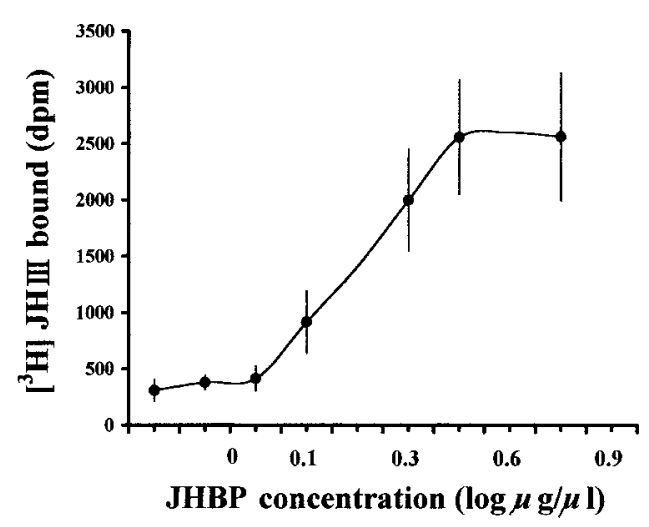

Fig. 3. Binding of $\left[{ }^{3} \mathrm{H}\right]$ JH III by locust haemolymph JHBP as a function of $\log$ JHBP concentration. Various concentrations of partially purified JHBP were incubated and assayed for binding with $\left[{ }^{3} \mathrm{H}\right] \mathrm{JH}$ III by the HAP assay. Data points represent the average of three separate determinations and vertical bars represent \pm SEM.

for representative curve). The sensitivity of the assay for $50 \%$ competition is about $0.12 \mathrm{pM} \mathrm{JH} \mathrm{III}$ per assay tube. To optimize the extraction of $\mathrm{JH}$ from samples, haemolymph of $N$. lugens was mixed with methanol as described in Materials and Methods and then marker $\left[{ }^{3} \mathrm{H}\right] \mathrm{JH}$ III was added. The mixture was extracted with hexane five times 


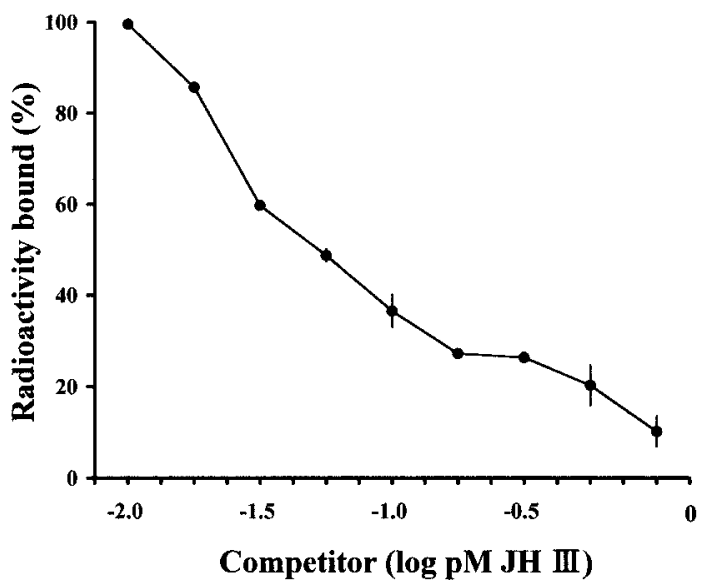

Fig. 4. Standard curve for the competition assay (see Materials and Methods). Displacement of $\left[{ }^{3} \mathrm{H}\right] \mathrm{JH}$ III as a function of $\log$ concentration of unlabeled JH III. Uncompeted binding was $4,400 \mathrm{dpm}$. Values shown are the means \pm SEM (three replicate samples in one experiment): where no error limits are shown they fall within experimental point symbols.

and radioactivity in each extract was counted. Efficiency of recovery of marker $\left[{ }^{3} \mathrm{H}\right] \mathrm{JH}$ III was determined to be $84 \%$ from five extractions. In all subsequent assays, five extractions were used. To determine whether lipid interacts with the binding assay, hexane extract of the haemolymph was added with marker $\left[{ }^{3} \mathrm{H}\right] \mathrm{JH}$ III and loaded to a SepPak silica cartridge. It was found that the eluate with $1.5 \mathrm{ml}$ hexane : ether $(9: 1$ by volume; see Materials and Methods) contained nearly $100 \%$ of the JH III. This indicates that purification by use of silica cartridges was necessary to prevent the lipid components of the haemolymph from interfering in the competitive binding.

The apparent JH III content of each sample assayed was determined from a standard curve generated at the same time. JH III titer was calculated according to sample size, sample dilution (selected to produce approximately $50 \%$ competition), and efficiency of extraction into hexane.

\section{Competitive binding assay determination of the amount of $\mathrm{JH}$ in biological samples}

JH III titer developmental profiles were similar in the brachypterous and macropterous last stadium nymphs (Fig. 5), as determined by the competitive binding assay. JH III levels were high during the first and second days of the stadium (85-95 nM) and dropped to very low levels on day 3 (24-34 nM). Mean JH III titers of the presumptive

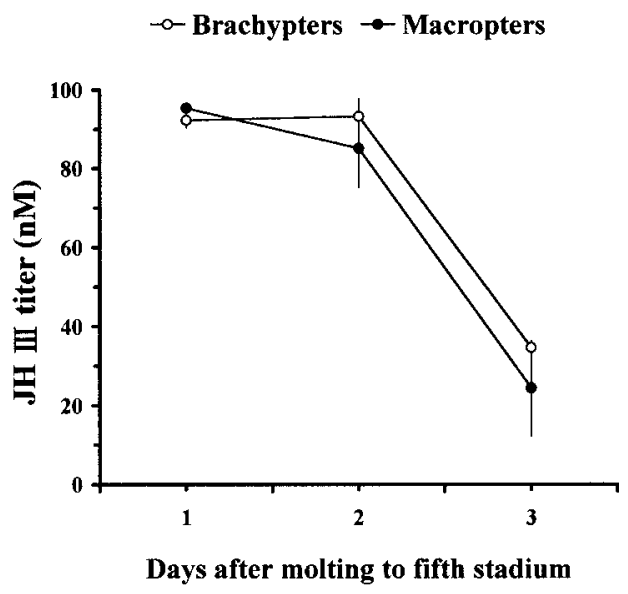

Fig. 5. Haemolymph JH III titer in the brachypterous $(O)$ and macropterous ( $\bullet$ fifth (final) stadium nymphs of $N$. lugens as determined by competitive binding assay. Each $\mathrm{JH}$ assay was done for pooled haemolymph from 100 to 188 animals. Twenty to $40 \mu \mathrm{l}$ was processed for the $\mathrm{JH}$ competition assay as described in Materials and Methods. Competition values are the averages of three determinations. Differences between the two wing forms were not significant ( $p>0.05$; Mann-Whitney $U$ test).

brachypterous and macropterous fifth stadium nymphs were not significantly different $(p>0.05$; Mann-Whitney $U$ test).

Changes of $\mathrm{JH}$ titer in the haemolymph during the early adult stage of brachypters and macropters, respectively emerged from brachypterous and macropterous pure lines of $N$. lugens are presented in Figs. 6B (females) and 7 (males). JH III titers in brachypters and macropters ranged from 27 to $94 \mathrm{~nm}$ in all wing forms and sexes. The mean JH III titers in the brachypters were significantly higher than those in the macropters ( $p \leqq 0.001$; MannWhitney $U$ test), except at day 1 in males (Fig. 7).

Patterns of terminal oocyte growth differed between brachypters and macropters (Fig. 6A). Brachypter females had significantly longer terminal oocytes than macropter on days 2, 3 and 4 ( $p \leqq 0.001$, Mann-Whitney $U$ test), but not on day 1 ( $p>0.05$, Mann-Whitney $U$ test). The terminal oocytes of female brachypters approached maximum length at day 3 after eclosion.

\section{DISCUSSION}

The present study revealed the presence of JH III in $N$. lugens, but the levels were very close to the detection limit of the method (Bergot et al., 1981) 

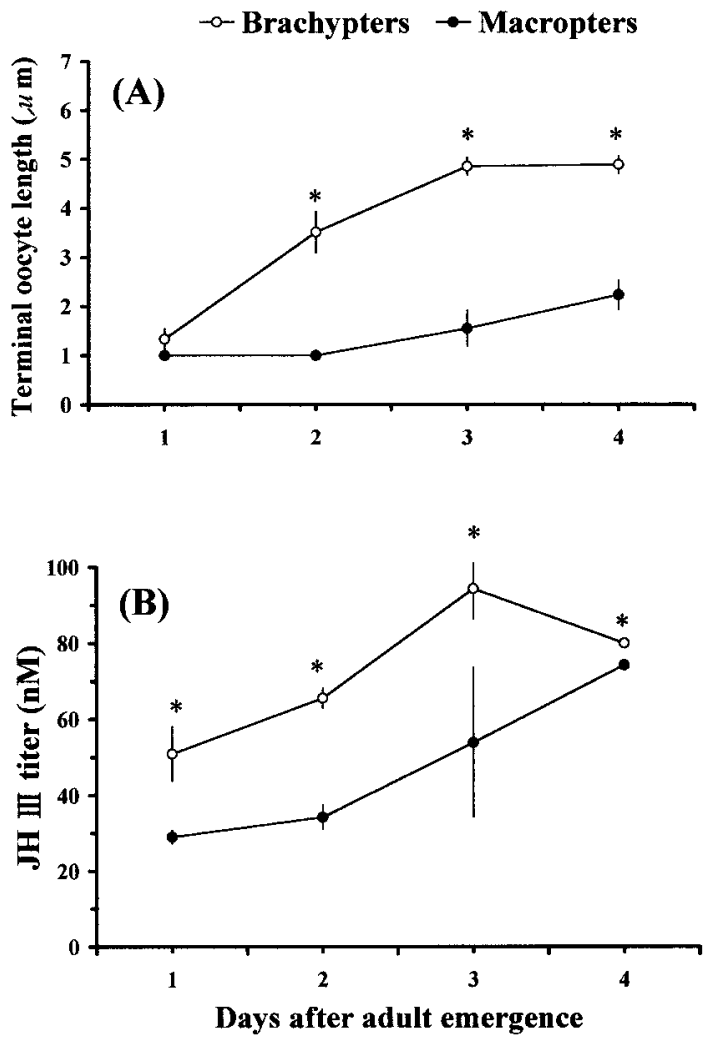

Fig. 6. Length of terminal oocyte (A) and haemolymph JH III titer (B) in the brachypterous $(O)$ and macropterous $(\bullet)$ females of $N$. lugens as determined by competitive binding assay. Each JH assay was done for pooled haemolymph from 40 to 100 animals. Twenty to $40 \mu \mathrm{l}$ were processed for the $\mathrm{JH}$ competition assay as described in Materials and Methods. Competition values are the averages of three determinations. Asterisks indicate statistical difference between the two wing forms of the same age ( $p \leqq 0.001$, Mann-Whitney $U$ test).

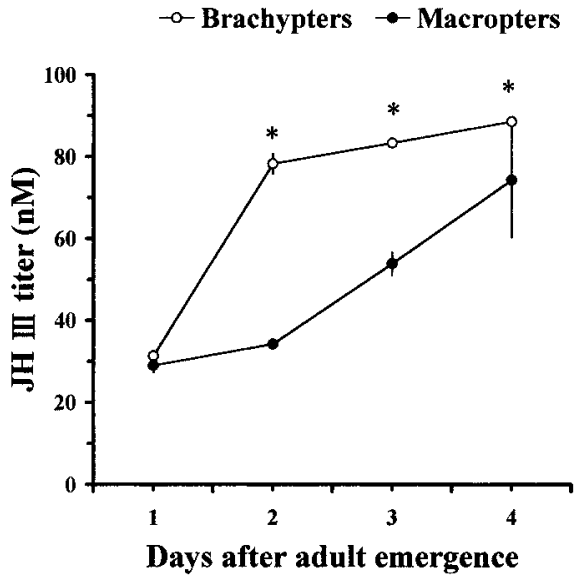

Fig. 7. Haemolymph JH III titer in the brachypterous $(\mathrm{O})$ and macropterous ( $\bullet$ males of $N$. lugens as determined by competitive binding assay. (See Fig. 6 for details). which prevented us from making an accurate calculation of the JH titers, although considerable efforts have been made to unequivocally confirm the identity of JH III (Figs. 1 and 2). The identification of $\mathrm{JH}$ III as the predominant $\mathrm{JH}$ in both nymphs and adults of $N$. lugens is the first case of identification of the natural hormone among planthoppers. The absence or no detection of other JH homologues (i.e., JH II, JH I, JH O and 4-MeJH I) in $N$. lugens is consistent with data from other non-lepidopterans (Schooley et al., 1984). JH III has been found to be the predominant homologue among 100 insect species representing at least ten insect orders studied by Baker (1990). Hardie et al. (1985) also identified JH III in the whole body extracts of the homopterans, Megoura viviae and Aphis fabae.

JHBP in the haemolymph of L. migratoria has been found to have an exceptionally high affinity for JH III (Trowell, 1992). It has been reported that JHBP in the haemolymph of L. migratoria has a $K_{\mathrm{d}}$ of $1.4 \mathrm{nM}$ for $\left[{ }^{3} \mathrm{H}\right] 10 \mathrm{R}$ JH III (Braun et al., 1995) and $K_{\mathrm{d}}$ of $3.6 \mathrm{~nm}$ for 10R-JH III (Koopmanschap and de Kort, 1988). The results presented in this paper clearly show that purified haemolymph JHBP of precocene-treated adult L. migratoria gives rapid and stable binding to $\left[{ }^{3} \mathrm{H}\right] \mathrm{JH}$ III (Fig. $3)$. It has also been observed that various concentrations of unlabeled JH III competed very effectively with $\left[{ }^{3} \mathrm{H}\right] \mathrm{JH}$ III, showing $50 \%$ displacement at $0.12 \mathrm{pm}$ (31 pg) (Fig. 4). By comparing the characteristic displacement of a JH III standard to the displacement of haemolymph samples of $N$. lugens, following standardization of the assay to optimize binding, we were able to quantify the JH III of the samples.

Studies revealed that $N$. lugens are highly sensitive to exogenous treatment of JH III by being affected in metamorphosis and wing development (Ayoade et al., 1999; Bertuso, A.G., unpublished). This suggests that JH III can act as the sole JH of $N$. lugens. During the fifth (final) nymphal stadium, the JH titer was high at the beginning of the stadium (days 1 and 2) and dropped to a low point at day 3 (Fig. 5). Our result is in accordance with the classical scheme of hormonal control of metamorphosis (also known as high-low-no hypothesis) at this time (Schneiderman and Gilbert, 1964). The decline of $\mathrm{JH}$ titer on day 3 is possibly due to $\mathrm{CA}$ shut off, when nymphs reach the critical weight at 
this stadium. Inactivation of the CA may be followed shortly after by a rise in the level of JH-specific esterases in the haemolymph, which rapidly inactivate the remaining JH (Hammock, 1985).

It has been believed that higher $\mathrm{JH}$ titer determines the development of the brachypterous (or apterous) vs. macropterous phenotype (Nijhout and Wheeler, 1982; Hardie and Lees, 1985). Direct JH level comparisons between presumptive wing morphs have only been conducted on Gryllus rubens and G. firmus (Zera et al., 1989; Zera and Tiebel, 1989; Zera and Tobe, 1990; Cisper et al., 2000). Haemolymph JH titers measured in the penultimate and last stadium of $G$. rubens ranged from 15 to $40 \mathrm{~nm}$ and from 1 to $10 \mathrm{~nm}$, respectively. However, no large differences in JH titers were observed between the brachypterous and macropterous morphs of G. rubens (Zera et al., 1989). In $N$. lugens, subtle JH III titer differences between the presumptive brachypterous and macropterous fifth stadium nymphs were observed (Fig. 5). The functional significance of these titer differences in wing morph determination is unclear. However, previous experiments have shown that wing development may be redirected from macropters to brachypters in $N$. lugens when JH III is applied during the third and fourth (penultimate) stadia, suggesting that a high $\mathrm{JH}$ titer during these stadia may determine the brachypterous phenotype (Iwanaga and Tojo, 1986; Ayoade et al., 1996a, b, 1999). Determination of $\mathrm{JH}$ titers during these stadia is deemed necessary for a clear understanding of the regulatory role of $\mathrm{JH}$ III in wing polymorphism in $N$. lugens.

$\mathrm{JH}$ has been shown to control ovarian maturation in insects by regulating processes of vitellogenin synthesis by the fat body, separation of a new follicle from the germanium, previtellogenic growth of the oocyte and vitellogenesis (reviewed by Nijhout, 1994). Our results clearly showed that terminal oocyte length (Fig. 6A) and JH titer (Fig. 6B) were positively correlated, implicating $\mathrm{JH}$ as a regulator of oocyte growth in $N$. lugens. Also in females, JH titer in the brachypters rose early on days 1,2 and 3 compared to the macropters, corresponding with the fact that ovarian maturation is faster in brachypters relative to macropters. Vitellogenin in $N$. lugens, identified by electrophoresis, first appeared in female haemolymph at day 1 or 2 and day 3 of adult eclosion in the brachypters and macropters, respectively (Inada, M., unpublished), suggesting that JH III stimulates vitellogenin synthesis and vitellogenesis. A recent study showed that the $\mathrm{JH}$ titer of G. firmus was higher in shortwinged females compared with flight-capable females during the first week of adulthood, in which greater ovarian growth in short-winged females was also shown to be due to an elevated JH titer (Cisper et al., 2000). Short-winged morphs also have greater ovarian mass than the long-winged morphs of G. rubens (Zera and Rankin, 1989; Mole and Zera, 1994). JH titers were also observed to be higher in the brachypter vs. macropter males at days 2, 3 and 4 of adulthood (Fig. 7). Our present finding in $N$. lugens, specifically, the different trait of $\mathrm{JH}$ titers between the two wing-forms, is in keeping with the migratory syndrome influenced by JH (Dingle, 1985; Fairbairn, 1994) and the apparent flight-capability vs. fecundity trade-off (Harrison, 1980) observed in wing dimorphic species.

Mean JH III titers of female (28-86 nM) and male (30-88 nм) $N$. lugens during early adulthood (Fig. 6B) were lower than the JH III titers of 5-d old female and male L. migratoria (166 nM and 228 nM, respectively; Glinka et al., 1995). Both experiments used locust haemolymph JHBP for the competitive protein binding assay. Tobe et al. (1984), Sevala et al. (1999) and Engelmann and Mala (2000) reported high JH titers in adults of Diploptera punctata $(6.0 \mu \mathrm{M})$, Blatella germanica $(0.6 \mu \mathrm{M})$ and Leucophaea maderea $(1.5 \mu \mathrm{M})$, respectively. The differences in the JH titers among species are unclear. Perhaps characterization of hormone receptors will provide answers to this puzzle.

\section{ACKNOWLEDGEMENTS}

This work was supported in part by a grant from the Ministry of Education, Science, Sports and Culture of Japan (No. 13460023). We would like to thank Dr. Hajime Sugie, National Institute of Agro-Environmental Sciences, Tsukuba, Ibaraki, for help in the GC/MS analysis of samples. We also thank Dr. Seiji Tanaka, Laboratory of Insect Life-Cycles and Physiology, Institute of Insect and Animal Sciences, Tsukuba, Ibaraki, for the nymphs of Locusta migratoria. Thanks are due to Dr. Sunao Morooka, Saga University, Saga, for providing the Nilaparvata lugens pure lines and for his valuable suggestions to the present study.

\section{REFERENCES}

Ayoade, O., S. Morooka and S. Tojo (1996a) Induction of macroptery, precocious metamorphosis, and retarded 
ovarian growth by topical application of Precocene II with evidence of its non-systemic allaticidal effects in the brown planthopper, Nilaparvata lugens. J. Insect Physiol. 42: 529-540.

Ayoade, O., S. Morooka and S. Tojo (1996b) Metamorphosis and wing formation in the brown planthopper, Nilaparvata lugens, after topical application of Precocene II. Arch. Insect Biochem. Physiol. 32: 485-491.

Ayoade, O., S. Morooka and S. Tojo (1999) Enhancement of short wing formation and ovarian growth in the genetically defined macropterous strain of the brown planthopper, Nilaparvata lugens. J. Insect Physiol. 45: 93-100.

Baker, F. C. (1990) Techniques for identification and quantification of juvenile hormones and related compounds in Arthropods. In Morphogenetic Hormones in Arthropods: Discovery, Synthesis, Metabolism, Evolution, Mode of Action, and Techniques (A. P. Gupta ed.). Rutgers University Press, New Brunswick, pp. 389-453.

Bergot, B. J., M. Ratcliff and D. A. Schooley (1981) Method for quantitative determination of the four known juvenile hormones in insect tissue using gas chromatography-mass spectroscopy. J. Chromatogr. 204: 231-244.

Bergot, B. J., D. A. Schooley, G. M. Chippendale and C.-M. Yin (1976) Juvenile hormone titer determinations in the southwestern corn borer, Diatraea grandiosella, by electron capture-gas chromatography. Life Sci. 18: 811-820.

Bowers, W. S., P. A. Marsella and P. H. Evans (1983) Identification of an hemipteran juvenile hormone: in vitro biosynthesis of JH III by Dysdercus fasciatus. J. Exp. Zool. 228: 555-559.

Braun, R. P., G. E. Edwards, K. J. Yagi, S. S. Tobe and G. R. Wyatt (1995) Juvenile hormone binding components of locust fat body. Arch. Insect Biochem. Physiol. 28: 291309.

Chinzei, Y., B. N. White and G. R. Wyatt (1982) Vitellogenin mRNA in locust fat body: Identification, isolation and quantitative changes induced by juvenile hormone. Can. J. Biochem. 60: 243-251.

Cisper, G., A. J. Zera and D. W. Borst (2000) Juvenile hormone titer and morph-specific reproduction in the wingpolymorphic cricket, Gryllus firmus. J. Insect Physiol. 46: 585-596.

Darrouzet, E., B. Mauchamp, G. D. Prestwich, L. Kerhoas, I. Ujvary and F. Couillaud (1997) Hydroxy juvenile hormones biosynthesized by locust corpora allata in vitro. Biochem. Biophys. Res. Commun. 240: 752-758.

Davey, K. G. (2000) The modes of action of juvenile hormones: some questions we ought to ask. Insect Biochem. Molec. Biol. 30: 663-669.

Dingle, H. (1985) Migration and life histories. In Migration: Mechanisms of Adaptive Significance (M. A. Rankin ed.). Contributions to Marine Science (Suppl.), Port Aransas, Taxas, pp. 27-44.

Englemann, F. and J. Mala (2000) The interactions between juvenile hormone $(\mathrm{JH})$, lipophorin, vitellogenin, and JH esterases in two cockroach species. Insect Biochem. Molec. Biol. 30: 793-803.

Fairbairn, D. J. (1994) Wing dimorphism and migratory syndrome: correlated traits for dispersal tendency in wing di- morphic insects. In Proceedings of Memorial and International Symposium on Dispersal Polymorphism in Insects, Its Adaptation and Evolution, Okayama University, Japan, pp. 143-152.

Gilbert, L. I., N. A. Granger and R. Michael Roe (2000) The juvenile hormones: Historical facts and speculations on future research directions. Insect Biochem. Molec. Biol. 30: 617-644.

Glinka, A. V., R. P. Braun, J. P. Edwards and G. R. Wyatt (1995) The use of a juvenile hormone binding protein for the quantitative assay of juvenile hormone. Insect Biochem. Molec. Biol. 25: 775-781.

Goodman, W., W. E. Bollenbacher, H. L. Zvenko and L. I. Gilbert (1976) A competitive binding protein assay for juvenile hormone. In The Juvenile Hormones (L. I. Gilbert ed.). Plenum Press, New York, pp. 75-95.

Hammock, B. D. (1985) Regulation of juvenile hormone titer: Degradation. In Comprehensive Insect Physiology, Biochemistry and Pharmacology (G. A. Kerkut and L. I. Gilbert eds.). Pergamon Press, New York, pp. 431-472.

Hardie, J., F. C. Baker, G. C. Jamieson and A. D. Lees (1985) The identification of an aphid juvenile hormone, and its titre in relation to photoperiod. Physiol. Entomol. 10: 297-302.

Hardie, J. and A. D. Lees (1985) Endocrine control of polymorphism: Endocrinology II. In Comprehensive Insect Physiology, Biochemistry and Pharmacology (G. A. Kerkut and L. I. Gilbert eds.). Pergamon Press, Toronto, pp. 441-491.

Harrison, R. G. (1980) Dispersal polymorphism in insects. Ann. Rev. Ecol. Syst. 11: 95-118.

Iwanaga, K. and S. Tojo (1986) Effects of juvenile hormone and rearing density on wing dimorphism and oocyte development in the brown planthopper, Nilaparvata lugens. J. Insect Physiol. 32: 585-590.

Koopmanschap, A. B. and C. A. D. de Kort (1988) Isolation and characterization of a high molecular weight JH-III transport protein in the hemolymph of Locusta migratoria. Arch. Insect Biochem. Physiol. 7: 105-118.

Kotaki, T. (1993) Biosynthetic products by heteropteran corpora allata in vitro. Appl. Entomol. Zool. 28: 242-245.

Kotaki, T. (1996) Evidence for a new juvenile hormone in a stink bugs Plautia stali. J. Insect Physiol. 42: 279-286.

Mauchamp, B., R. Lafont, M. Hardy and D. Jourdain (1979) Analysis of insect juvenile hormone by gas chromatography mass spectrometry: Problems of sample preparation and choice of detection procedure. Biomed. Mass Spect. 6: 276-281.

Mole, S. and A. J. Zera (1994) Differential resource consumption obviates a potential flight-fecundity trade-off in the sand cricket (Gryllus firmus). Funct. Ecol. 8: 573-580.

Morooka, S. and S. Tojo (1992) Maintenance and selection of strains exhibiting specific wing form and body colour under high density conditions in the brown planthopper, Nilaparvata lugens (Homoptera: Delphacidae). Appl. Entomol. Zool. 27: 445-454.

Nijhout, H. F. (1994) Insect Hormones. Princeton University Press, New Jersey. 267 pp.

Nijhout, H. F. and D. E. Wheeler (1982) Juvenile hormone and 
the physiological basis of insect polymorphism. Quart. Rev. Biol. 57: 109-133.

Numata, H., A. Numata, C. Takahashi, Y. Nakagawa, K. Iwatani, S. Takahashi, K. Miura and Y. Chinzei (1992) Juvenile hormone I is the principal juvenile hormone in a hemipteran insect, Riptortus clavatus. Experientia 48: 606-610.

Rankin, M. A. and L. M. Riddiford (1977) Hormonal control of migratory flight in Oncopeltus fasciatus: The effect of the corpous cardiacum, corpus allatum, and starvation on migration and reproduction. Gen. Comp. Endocr. 33: 309-321.

Rankin, M. A. and L. M. Riddiford (1978) Significance of haemolyph juvenile hormone titer changes in timing of migration and reproduction in adult Oncopeltus fasciatus. J. Insect Physiol. 24: 31-38.

Rembold, H. and B. Lackner (1985) Convenient method for the determination of picomole amounts of juvenile hormone. J. Chromatogr. 323: 355-361.

Riddiford, L. M. (1994) Cellular and molecular actions of juvenile hormone: General considerations and premetamorphic actions. Adv. Insect Physiol. 24: 213-274.

Roberts, P. E. and G. R. Wyatt (1983) Juvenile hormone binding by components of fat body cytosol from vitellogenic locust. Molec. Cell. Endoc. 31: 53-69.

Schneiderman, H. A. and L. I. Gilbert (1964) Control of growth and development in insects. Science 143: 325333.

Schooley, D. A., F. C. Baker, L. W. Tsai, C. A. Miller and G. C. Jamieson (1984) Juvenile hormones, O, I and II exist only in Lepidoptera. In Biosynthesis, Metabolism and Mode of Action of Invertebrate Hormones (J. Hoffman and M. Porchet eds.). Springer, Berlin, pp. 373-383.

Sevala, V., S. Shu, S. B. Ramaswamy and C. Schal (1999) Lipophorin of female Blatella germanica $(\mathrm{L})$ : characterization and relation to haemolymph titers of juvenile hormone and hydrocarbons. J. Insect Physiol. 45: 431-441.

Staal, G. B. (1975) Insect growth regulators. Ann. Rev. Entomol. 20: 417-460.

Staal, G. B. (1986) Anti juvenile hormone agents. Ann. Rev. Entomol. 31: 391-429.

Strambi, C., A. Strambi, M. De Reggi and M. Delaage (1984)
Radioimmunoassay of juvenile hormones. State of the methods and recent data on validation. In Biosynthesis, Metabolism and Mode of Action of Invertebrate Hormones (J. Hoffman and M. Porchet eds.). Springer, Berlin, pp. 355-362.

Tobe, S. S., B. A. Stay, F. C. Baker and D. A. Schooley (1984) Regulation of juvenile hormone titer in the adult female cockroach Diploptera punctata. In Biosynthesis, Metabolism and Mode of Action of Invertebrate Hormones (J. Hoffman and M. Porchet eds.). Springer, Berlin, pp. 397406.

Trowell, S. C. (1992). High affinity juvenile hormone carrier proteins in the haemolymph of insects. Comp. Biochem. Physiol. 103B: 795-807.

Wilkins, R. M. and C. H. Sim (1983) Toxicity of precocenes and analogs to the rice brown planthopper. In Proceedings of the 10th International Conference on Plant Protection in Brighton. Lavenham, Suffolk, Lavenham, p. 279.

Yin, C. M., B.-X. Zou, M. Jiang, M.-F. Li, W. Qin, T. L. Potter and J. G. Stoffolano (1995) Identification of juvenile hormone III and methyl farnesoate secreted by the corpus allatum of Phormia regina (Meigen) in vitro and function of $\mathrm{JHB}_{3}$, either applied alone or as part of a juvenoid blend. J. Insect Physiol. 40: 283-292.

Zera, A. J. and M. A. Rankin (1989) Wing dimorphism in Gryllus rubens: Genetic basis of morph determination and fertility differences between morphs. Oecologia 80: 249-255.

Zera, A. J., C. Strambi, K. C. Tiebel, A. Strambi and M. A. Rankin (1989) Juvenile hormone and ecdysteroid titers during critical periods of wing morph determination in Gryllus rubens. J. Insect Physiol. 35: 501-511.

Zera, A. J. and K. C. Tiebel (1989) Differences in juvenile hormone esterase activity between presumptive macropterous and brachypterous Gryllus rubens: Implications for the hormonal control of wing polymorphism. J. Insect Physiol. 35: 1-17.

Zera, A. J. and S. S. Tobe (1990) Juvenile hormone-III biosynthesis in presumptive long-winged and short-winged Gryllus rubens: Implications for the endocrine regulation of wing dimorphism. J. Insect Physiol. 36: 271-280. 